\title{
Estudio de los pavimentos de túneles carreteros: ventajas de los pavimentos de hormigón frente a los bituminosos
}

\author{
Study of motorway tunnel pavements: advantages of concrete against asphalt pavements
}

Fecha de entrega: 6 de febrero 2018

Fecha de aceptación: 7 de mayo 2018

\section{Jesús Díaz y Fernando Hacar ${ }^{2}$}

${ }^{1}$ IECA Tecnología, Instituto Español del Cemento y sus Aplicaciones, C/ José Abascal, 53 - 1º, 28003 Madrid, España, jdiaz@ieca.es 2 Ingeniero Técnico de Obras Públicas, Madrid, España, fhacar@gmail.com

Considerando tanto drenaje de un vertido accidental de una mercancía peligrosa sobre la calzada, asi como el fuego de dicho vertido con afección a la capa de rodadura, se realiza un estudio comparado de los pavimentos de hormigón y pavimentos bituminosos para túneles de carretera (con capas de rodadura de hormigón o bituminosas). Según se concluye en el presente trabajo, en túneles carreteros en los que se autorice el paso de mercancías peligrosas, la consideración conjunta de ambos aspectos aconseja la ejecución de pavimentos con capa de rodadura de hormigón (pavimentos de hormigón) en túneles de más de $500 \mathrm{~m}$ de longitud.

Palabras clave: túnel de carretera, vertido accidental, pavimento de hormigón, pavimento bituminoso, fuego
Considering both drainage of an accidental spillage of a dangerous goods on the road, as well as the fire of that spill with affection to the surface layer, a comparative study is made of the concrete pavements and bituminous pavements for road tunnels (with surface layers of concrete or bituminous). As concluded in the present work, in road tunnels that authorize the passage of dangerous goods, the joint consideration of both aspects advises the execution of pavements with surface layers of concrete (concrete pavements) in tunnels of more than $500 \mathrm{~m}$ long.

Keywords: road tunnel, accidental spillage, concrete pavement, bituminous pavement, fire

\section{Introducción}

De forma general, el pavimento de una carretera está constituido por un conjunto de capas horizontales cuya función es transmitir a la explanada las solicitaciones del tráfico suficientemente amortiguadas para que puedan ser soportadas por ésta sin deformarse. Por otra parte, la capa de rodadura (bien sea de un material bituminoso o de hormigón) debe proporcionar una superficie de rodadura cómoda y segura para la circulación de los vehículos (Miró, 2006).

Tal vez el pavimento de los túneles puede ser considerado como una unidad diferenciada o un elemento independiente dentro del tramo de carretera del que forman parte. El pavimento que se disponga en un túnel debe estudiarse detenidamente, sin la necesidad de coincidir con el del resto de la carretera. En algunos países es usual la colocación de un pavimento en la carretera y otro diferente en todas las estructuras o túneles, resultando esta práctica perfectamente asumida por el conductor.

Seguidamente se presentan algunos estudios relativos a la discusión relativa al empleo de pavimentos de hormigón o pavimentos bituminosos en túneles.

Como veremos en los siguientes epígrafes, es interesante resaltar que mientras los sistemas de drenaje para vertidos accidentales son preceptivos en túneles de más de $500 \mathrm{~m}$ (en Europa y en España) ó 300 m (en Francia) en los que se permita el paso de mercancías peligrosas, en España, por razones de fuego, los pavimentos de hormigón (con capa de rodadura de hormigón) sólo son preceptivos en túneles de más de $1 \mathrm{~km}$ (tanto si se autoriza o no el paso de mercancías peligrosas). 


\section{Pavimentos de hormigón y pavimentos bituminosos}

Algunos autores (e.g. del Val Melús et al., 2007; de Lathawer, 2007) señalan que el empleo de pavimentos bituminosos no produce un significativo adverso impacto en la seguridad en caso de incendio y por ello -según los citados autores- pueden ser empleados dichos pavimentos.

Por el contrario, estudios como el realizado por el GIDAI (2011) apuntan en sentido contrario. Las probetas de hormigón no se degradan ni a las altas temperaturas ensayadas superiores a $500^{\circ} \mathrm{C}$, generan poco humo (prácticamente vapor de agua) permitiendo cierta visibilidad, no entran en ignición y emite reducidas cantidades de gases tipo $\mathrm{CO}_{2}, \mathrm{CO}, \mathrm{SO}_{2}$ o $\mathrm{CH}_{4}$ (metano).

Posteriormente, el Instituto Español del Cemento y sus Aplicaciones IECA, contando con la colaboración de la Asociación Profesional de Técnicos de Bomberos APTB, realizan unas modelizaciones de diferentes incendios (APTB-IECA, 2013), recomendando el empleo de pavimentos de hormigón (capa de rodadura de hormigón) en todos los túneles por el mayor nivel de seguridad que proporciona en el caso de un incendio provocado por cualquier trágico accidente. Frente a otras soluciones, se reduce la emisión de humos y gases tóxicos, no aumenta la carga de fuego, no es inflamable, por lo que no contribuyen a la rápida extensión del fuego, y se mantiene integro a las temperaturas usualmente alcanzadas, lo que permite el acceso de los equipos profesionales de extinción y salvamento.

Seguidamente se transcriben algunos párrafos del citado estudio de la APTB-IECA (2013):

"Cuando la potencia del incendio supera los $30 \mathrm{MW}$ las llamas pueden comenzar a extenderse a ambos lados del vehículo por debajo de la bóveda si el sistema de evacuación de humos no puede absorber el volumen de gases generados. Las llamas pueden alcanzar varias decenas de metros en ambas direcciones. Como consecuencia la radiación emitida por las llamas aumentará alcanzando a nivel del suelo los $40 \mathrm{~kW} / \mathrm{m}^{2}$ y conseguirá en pocos segundos que la temperatura del pavimento llegue a los $300^{\circ} \mathrm{C}$. A partir de esas temperaturas es cuando los dos tipos de pavimento empiezan a comportarse de maneras muy diferentes.
Los pavimentos a base de mezclas bituminosas por tener un componente combustible como es el betún tendrán un comportamiento diferente durante un incendio. Cuando las temperaturas se aproximan a los $300^{\circ} \mathrm{C}$ las mezclas bituminosas comienzan a desprender humo y gases pero no comienzan su ignición hasta que la temperatura no llega a los $485^{\circ} \mathrm{C}$. En ese instante el pavimento empieza a emitir unas llamas que se mantendrán mientras quede betún por quemar y mientras la radiación sea superior a los $40 \mathrm{~kW} / \mathrm{m}^{2}$.

La combustión del betún, a pesar de representar un aporte de calor pequeño comparado con la combustión de la carga del vehículo pesado, representa una fuente de emisión de calor que incide en la zona inferior de la carga del vehículo, y acelerándolo. El aporte de calor por la zona inferior facilita que la carga entre en combustión generalizada mucho antes y de forma más violenta produciendo el aumento de potencia.

...

Otro factor fundamental para la extensión del incendio será la cercanía que haya con otros vehículos detenidos en el túnel. Cuando la potencia del incendio sobrepasa los $50 \mathrm{MW}$ el incendio se puede extender a vehículos pesados que están a más de $30 \mathrm{~m}$ debido a su mayor altura y cercanía a las llamas y gases que circulen por la bóveda. Ese efecto puede atrapar a los bomberos entre dos fuegos ya que si tienen que acercarse a menos de 20 m para realizar la proyección del agua se pueden formar nuevos focos de incendio por detrás de ellos. Por lo tanto si se evita el aumento de potencia debido a la combustión baja la seguridad de los equipos de extinción."

\section{Normativa}

En España, el Real Decreto 635 (2006) (que es la transposición de la Directiva (2004) de la Unión Europea) señala que en túneles de más de $1 \mathrm{~km}$ se ha de emplear pavimentos de hormigón [se sobreentiende que es con capa de rodadura de hormigón]: La principal ventaja del empleo de este tipo de pavimento se pone de manifiesto por su mejor comportamiento ante el fuego (Resolución, 2012).

Por lo que en lo referente al pavimento indica: 


\section{Anexo I: 2.3.1 Pavimento:}

Salvo razones debidamente justificadas, en túneles de más de 1000 metros se empleará pavimento de hormigón [se sobreentiende que es con capa de rodadura de hormigón] con aditivos coloreantes para que proporcionen suficiente contraste con las marcas viales.

El detalle que introduce el texto del Real Decreto 635 (2006) relativo al contraste de las marcas viales es recordatorio en el sentido que hay que logar el adecuado contraste de la marca vial blanca -color empleado en España- sobre la superficie clara del hormigón.

Por otra parte, los ensayos sistemáticos relativos a vertidos accidentales en túneles que realizó el Centro de Estudios de Túneles CETU (www.cetu.developpement-durable. gouv.fr) en 1994 mostraron, entre otros aspectos, que, como quedó probado en el ensayo del túnel de Grand Mare, Francia, el comportamiento de un pavimento con capa de rodadura drenante era contraproducente. Como consecuencia de ello, la Circular Interministerial Francesa (CI, 2006) prohíbe utilizar mezclas drenantes (en capas de rodadura) en túneles dado el mal comportamiento que muestran dichas capas en caso de vertidos accidentales sobre la calzada. Se tiene entonces que CI (2006) anula a la CI (2000) a excepción del Anexo 2, que se mantiene en vigor (annexe $n^{\circ} 2$.- Instruction technique relative aux dispositions de securite dans les nouveaux tunnels routiers: Conception et Exploitation).

\subsection{3 - Revêtement des chaussées:}

Les couches de roulement drainantes sont proscrites à l'intérieur des tunnels à plus de $50 \mathrm{~m}$ des têtes. Si une telle couche de roulement est utilisée aux abords du tunnel, le changement sera effectué dans une zone couverte afin d'éviter de créer, en cas de pluie, un point singulier en termes d'adhérence ou de projections à l'entrée ou à la sortie du tunnel.

\section{Pavimentos de hormigón en túneles}

Como características más destacadas de los pavimentos de hormigón frente a otras soluciones, cabe resaltar el inferior coste total como ha quedado probado en varios pavimentos ya construidos. Si actualmente en España, los pavimentos de hormigón pueden resultar ligeramente más caros de construir que los pavimentos bituminosos o de un coste similar en algún caso, la reducción en los gastos de mantenimiento y conservación, que son casi nulos, hace que los costes totales sean mucho más reducidos a los pocos años. En la práctica en España se ha considerado que el pavimento de hormigón tenía un coste nulo y cuando llegaba al final de su vida útil (muy variable desde 20 a 40 años dependiendo de las características del hormigón, del tráfico circulante y del espesor de losa), se demuele o reconstruye con un refuerzo bituminoso dispuesto encima (Díaz, 2016). Y esto sin considerar los costes que para el usuario suponen los atascos, cortes de carril u operaciones de mantenimiento. Igual sucede si se analiza todo el ciclo de vida del firme o el coste del ciclo de vida del firme (pavimento) en su conjunto y no solo el coste constructivo, pues la solución pavimento de hormigón resulta mucho más ventajosa.

Entre las citadas ventajas de este tipo de pavimentos para un túnel cabe destacar (Jofré et al., 2010a):

\section{Ventajas técnicas:}

- Los pavimentos de hormigón tienen una excelente capacidad estructural para soportar las acciones del tráfico pesado.

- Reducen la distancia de frenado frente a otras soluciones, lo que se traduce en mayor seguridad.

- Permiten fácil y económicamente una gran variedad de texturas.

- Evitan la aparición de roderas.

- Son capaces de resistir los ataques de carburantes y agentes químicos.

- Es posible reforzar cualquier carretera, incluso de rodadura bituminosa, con una capa delgada.

- La durabilidad es más elevada que la del resto de soluciones sin apenas mantenimiento.

\section{Ventajas medioambientales:}

- Se reduce el empleo de áridos de características más exigentes, permitiendo su empleo en otra parte. Las técnicas actuales de pavimento de hormigón extendido en dos capas (con una capa de rodadura muy delgada) o el pavimento funcional compuesto (base de hormigón con una capa delgada de rodadura bituminosa) permiten el empleo de áridos de menor calidad o reciclados en la 
capa inferior, al no estar sometidos directamente a las acciones del tráfico rodado.

- Permiten capturar $\mathrm{CO}_{2}$ e incluso otros elementos contaminantes contribuyendo así a mejorar el balance de emisiones a lo largo de su vida útil.

- No emiten lixiviados, ni volátiles.

- Es una técnica que permite el empleo de cementos con alto contenido de adiciones. Así se pueden incorporar subproductos industriales como escorias o cenizas volantes, a la vez que se reduce la cantidad de clínker, y se pueden reducir las emisiones durante su fabricación.

- Su elevada durabilidad hace que se evite el consumo de nuevos recursos en los refuerzos, a la vez que se reducen las emisiones de $\mathrm{CO}_{2}$ debidas a las operaciones de mantenimiento o refuerzo.

- En su puesta en obra se evita la exposición de los operarios a altas temperaturas, gases nocivos y partículas en suspensión.

- El pavimento es reciclable en su totalidad al final de su vida útil, permitiendo una actuación mucho más ecológica y posibilitando el ahorro de áridos naturales.

- En conjunto tiene un menor Coste del Análisis del Ciclo de Vida CACV.

\section{Ventajas económicas}

- Con un coste de construcción del mismo orden de magnitud que el de otras soluciones, la vida útil es mayor, y menores las necesidades de mantenimiento. Por ello, los costes totales (inversión inicial más mantenimiento y conservación) son siempre inferiores a los de otra solución.

- Debido a que las operaciones de mantenimiento son muy escasas, la afección que producen sobre los usuarios es mínima. Por ello son muy bajos los costes derivados de dichas operaciones, así como los debidos a retenciones, accidentes $\mathrm{u}$ otros.

Estas ventajas se ven parcialmente afectadas por la carencia de una red de subcontratistas que construyan este tipo de pavimentos, la mayor dificultad a la hora de reparar defectos constructivos o el mayor nivel de inversión inicial que requieren (no si se consideran todos los costes, incluidos los de mantenimiento y conservación).

En el caso de un túnel el menor número de operaciones de mantenimiento de este tipo de calzadas se verá favorecido dada la atención que requiere garantizar la durabilidad de la obra y lo costoso que resultan las reparaciones en el interior del mismo. Pero, de la misma manera que el número de actuaciones de mantenimiento en un firme rígido son muy inferiores, en el caso de realizarse, la reparación de defectos, especialmente en túneles será más costosa, incómoda, $\mathrm{y}$, sobre todo, algo más lentas a pesar de poder emplear hormigones de altas resistencias iniciales. De aquí la importancia de una correcta ejecución, ya que el mayor porcentaje de defectos suele deberse a ciertos errores durante la construcción.

Dado que una de las principales fuentes de defectos son las juntas (roturas de esquina, etc.), una interesante solución consiste en la supresión de dichas juntas del pavimento, adoptando soluciones continuas. La Figura 1 muestra el túnel Cointe en Lieja, Bélgica, el cual es unidireccional, de $1639 \mathrm{~m}$ de longitud, $116 \mathrm{~m}^{2}$ de sección transversal y fue abierto al tráfico el 2 de junio de 2000.

La disposición de un pavimento de hormigón armado continuo puede suponer la casi totalidad desaparición de las operaciones de mantenimiento en lo referente al firme, según han demostrado obras con más de 40 años como la autopista denominada "Y de Asturias" mostrada en la Figura 2 (autopistas A-8 y A-66, que enlazan Oviedo, Gijón y Avilés, España), con un total de $43 \mathrm{~km}$ de pavimento de hormigón armado continuo y dos calzadas de $12 \mathrm{~m}$ de plataforma. La vía se ha mantenido en perfectas condiciones de uso durante estos 40 años, requiriendo únicamente inversiones y reparaciones locales muy reducidas (137.292,88 € reales gastados únicamente), con una mínima afección al voluminoso tráfico que circula por ella (casi 55000 vehículos de IMD con un $8 \%$ de pesados).

En la construcción se pueden emplear extendedoras de encofrados deslizantes sobre orugas (hay equipos de trabajo hasta anchos máximos de $6 \mathrm{~m}, 8.5 \mathrm{~m}$, y los más grandes pueden llegar hasta $16 \mathrm{~m}$ ) o acabadoras de cilindro, que deslizan sobre carriles (Jofré et al., 2010b), pero nunca es aconsejable la realización manual con reglas vibrantes por cuestiones de regularidad (ver Figuras 3 y 4). 
Díaz, J. y Hacar, F. (2018). Estudio de los pavimentos de túneles carreteros: ventajas de los pavimentos de hormigón frente a los bituminosos. Obras y Proyectos 23, 87-95

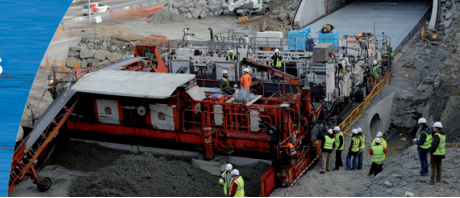

a)
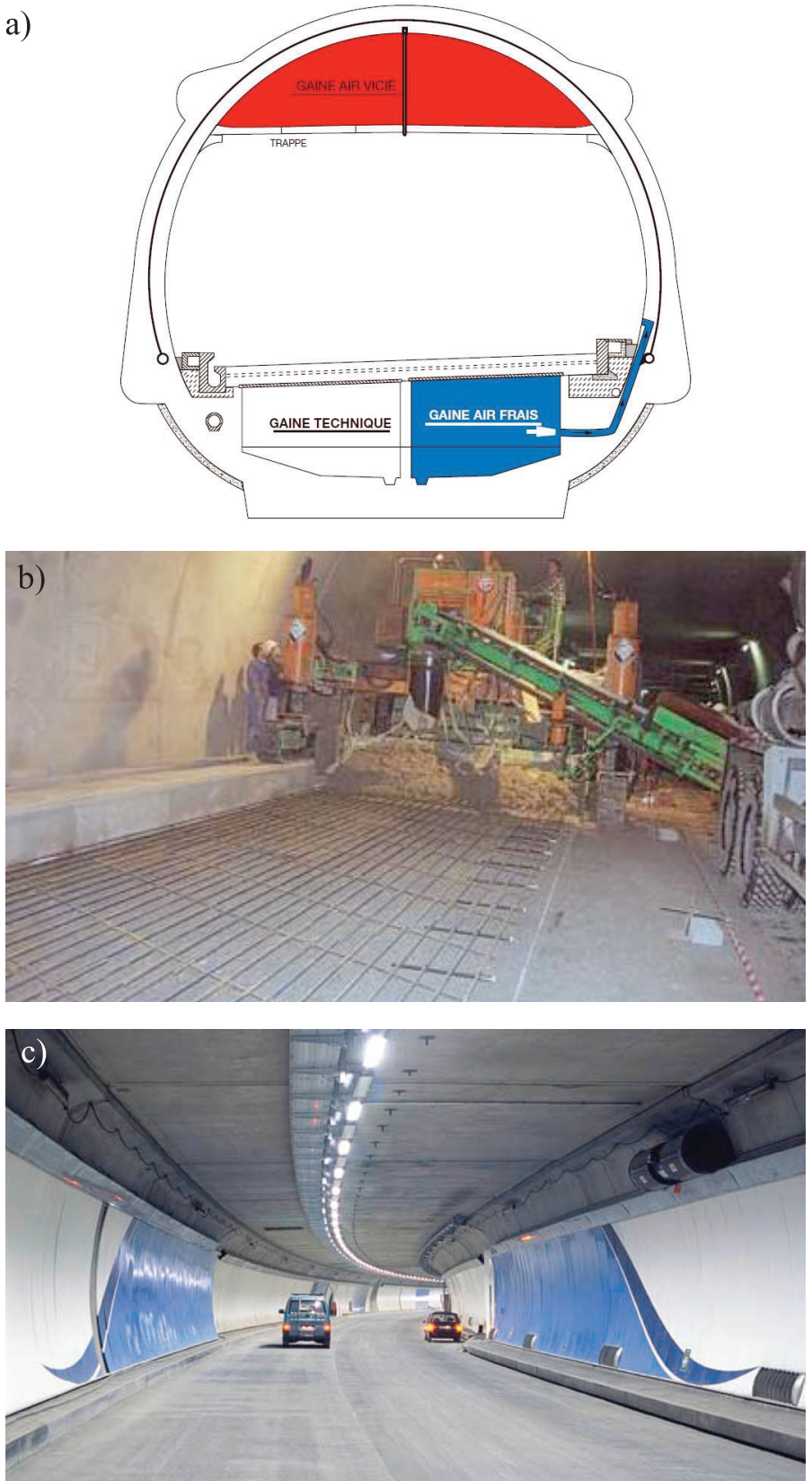

Figura 1: Túnel de Cointe (Lieja, Bélgica), abierto al tráfico el 2 de junio de 2000. Unidireccional, longitud 1639 m, sección 116 m2. a) Sección del túnel, b) ejecución de un pavimento de hormigón armado continuo y c) pavimento de hormigón armado continuo (Routes 83, 2003)

\section{Drenaje de vertidos accidentales}

\section{Ensayos de vertidos}

En los interesantes ensayos de vertidos del CETU de 1994, a los que nos hemos referido en líneas anteriores, se analizaron diferentes sistemas normalmente empleados en túneles para la evacuación de los fluidos que provengan de la calzada. Esos estudios incluyeron la revisión de los a)

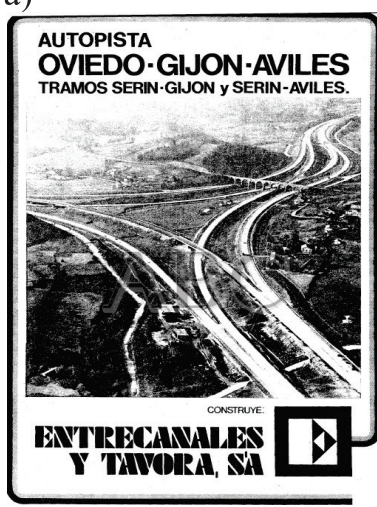

b)
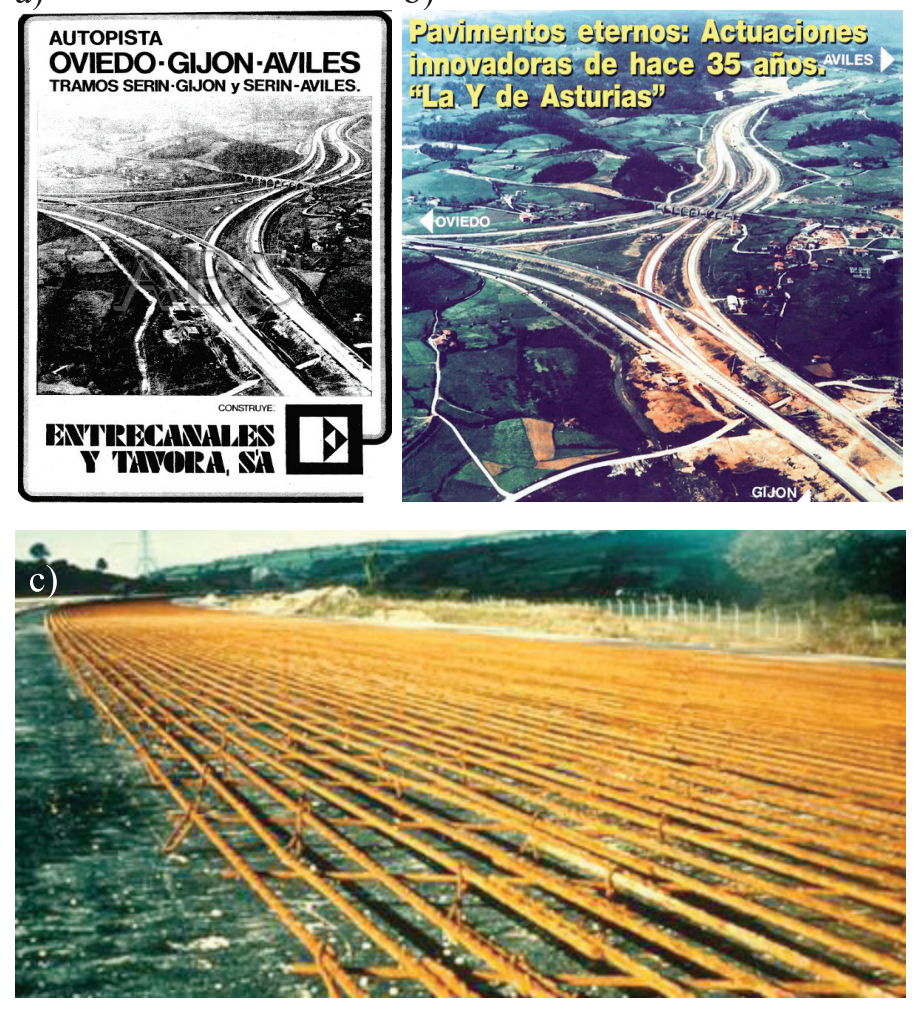

d)

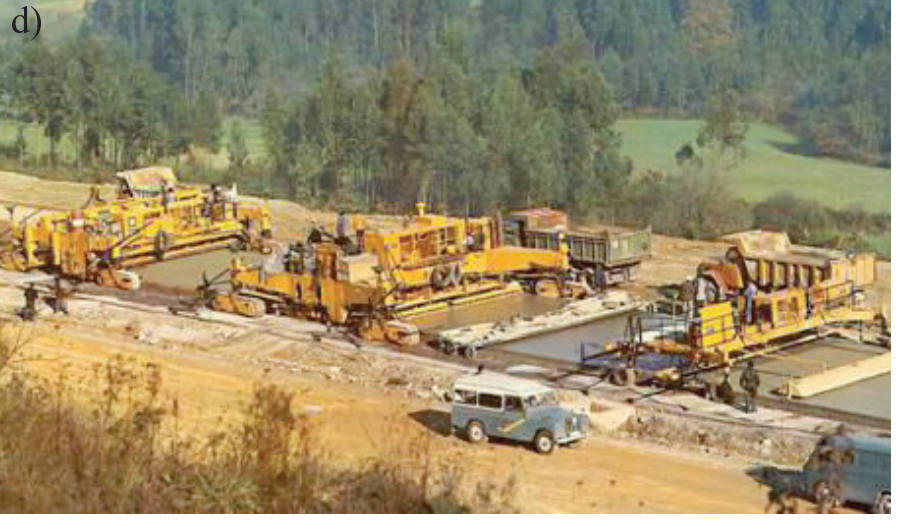

Figura 2: Pavimento de hormigón armado continuo de la autopista "Y Asturiana" en Asturias, España, a) periódico ABC de Madrid, 14 de febrero de 1976, b) panorámica de parte de la Autopista (Díaz, 2010), c) armaduras y d) colocación del hormigón

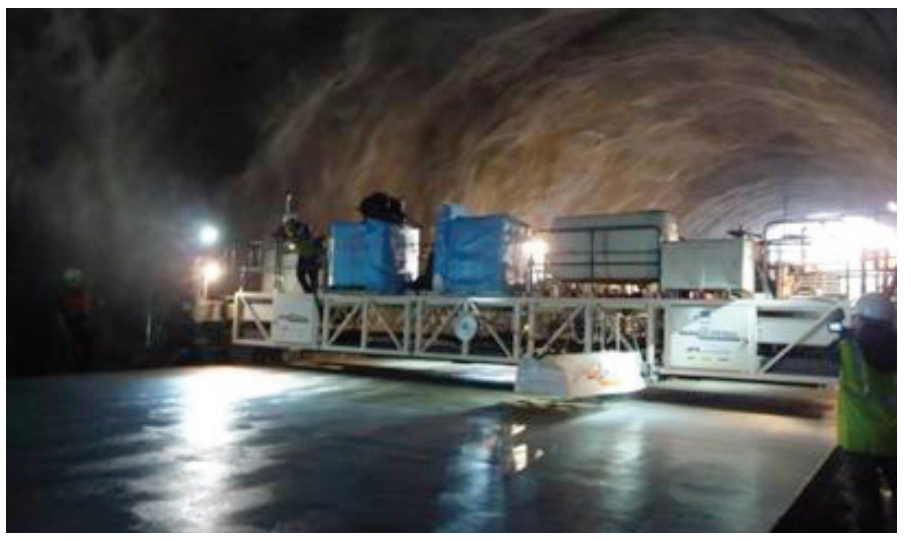

Figura 3: Ejecución del pavimento de hormigón en un túnel 

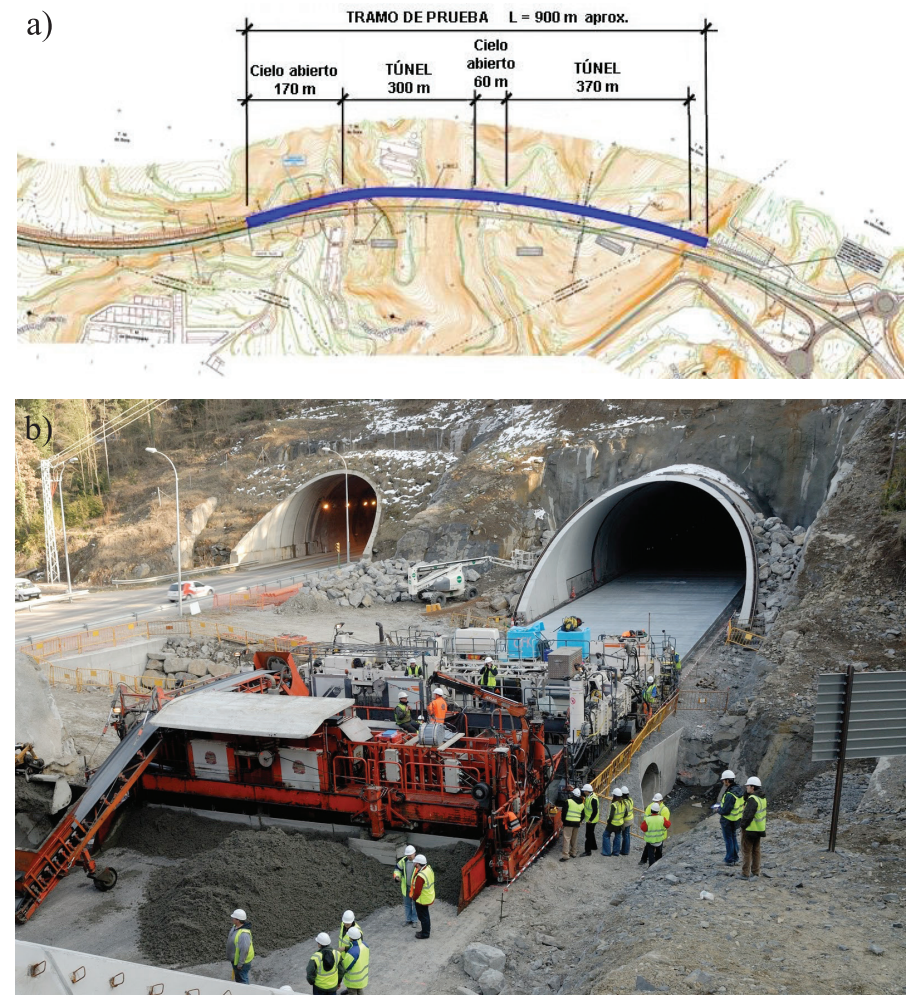

Figura 4: Realización del pavimento de hormigón, a) tramo de túnel entre Vic y Ripoll, Cataluña, España y b) desdoblamiento y acondicionamiento de la C-17 (FCC Construcción, 2010)

sistemas de recogida de líquidos inflamables en diversos tipos de instalaciones, tales como carreteras, aeropuertos, instalaciones petrolíferas, industrias químicas, etc., así como ensayos de la eficacia de los sistemas de cunetas y sifones cortafuegos, y ensayos de incendios realizados en una estación experimental por el Institut National de 1'Environnement Industriel et des Risques INERIS (Lacroix et al., 1995; Perard et al., 1996). Los ensayos pusieron de manifiesto que los sistemas de drenaje que tradicionalmente se empleaban en los túneles para los vertidos sobre la calzada (en muchos casos similares a los empleados al aire libre) no eran adecuados para hacer frente a uno de una sustancia en combustión, tal y como puede suceder con la rotura del racor o de un compartimento de una cisterna como se muestra en la Figura 5. Por un lado, al tener esos sistemas tradicionales poca eficiencia de captación, la superficie ocupada antes de tragar el fluido es muy elevada, y, por otra parte, esos sistemas no impedían que el fuego se propagase incluso por dentro de los colectores en los que podría producirse mezclas explosivas.

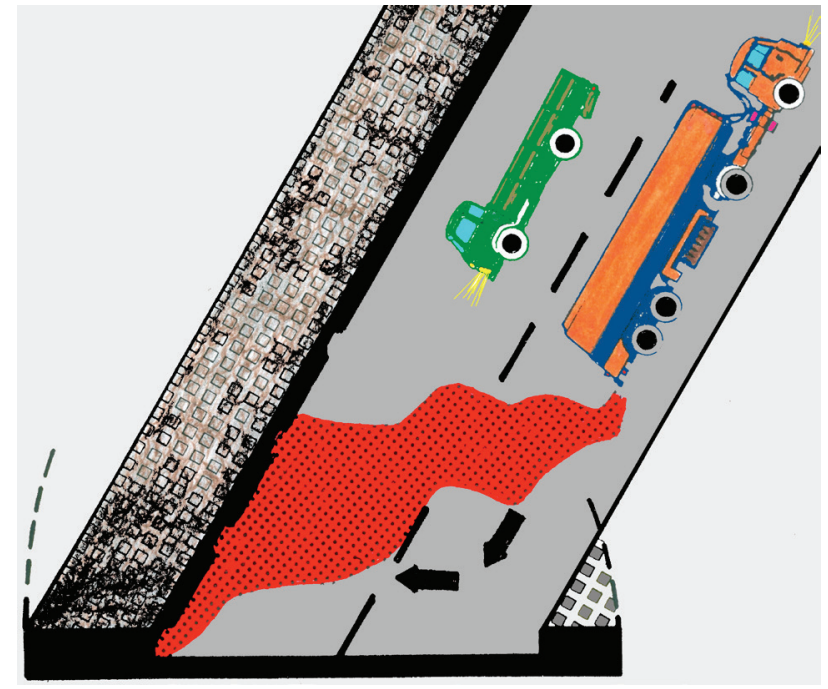

Figura 5: Primeros instantes del derrame de una cisterna

Los diferentes sistemas de evacuación de vertidos instalados en cada uno de los túneles que han sido ensayados por el CETU, representan una interesante muestra para deducir las características del sistema más adecuado desde el punto de vista de minimizar la superficie mojada. Igualmente, han demostrado que el funcionamiento de los sifones cortafuegos, ubicados a intervalos constantes a lo largo de las conducciones de evacuación, permiten reducir la propagación del fuego o de las explosiones a lo largo del túnel. Como consecuencia de lo indicado, la superficie del pavimento (la capa de rodadura) jugará un papel fundamental en caso de un vertido incendiado tanto desde el punto de vista del fuego como del drenaje de un vertido accidental. Seguidamente señalamos alguna normativa con el fin de destacar la relevancia y las medidas que se adoptan relativas a los vertidos accidentales en túneles.

\section{Normativa de Europa y de España}

En España, el Real Decreto 635 (2006) señala que en túneles de más de $500 \mathrm{~m}$ en los que se permita el paso de mercancías peligrosas se deberán instalar cauces que permitan el drenaje de líquidos tóxicos o inflamables con el fin de evitar que éstos se propaguen dentro del tubo o entre tubos. La Directiva Europea, aplicable a túneles de más de $500 \mathrm{~m}$ de longitud, se expresa en el mismo sentido.

\section{6.- Drenaje:}

2.6.1.- Si se permite el transporte de mercancías peligrosas, deberá haber alcantarillas de ranuras bien diseñadas, 
$u$ otros dispositivos, situadas dentro de las secciones transversales de los túneles, que permitan el drenaje de líquidos tóxicos e inflamables. Además, el sistema de drenaje deberá diseñarse y mantenerse de manera que se evite que el fuego y los líquidos inflamables y tóxicos se propaguen dentro de un tubo o entre tubos.

\subsection{2.- Al decidir si se permite el transporte de productos} peligrosos, basándose en un análisis de los riesgos correspondientes, se tendrá en cuenta si en los túneles existentes no se pueden cumplir tales requisitos o sólo se pueden cumplir con costes desproporcionados.

\section{Normativa de Francia}

La Circular Interministerial francesa del año 2000 prescribe que dicho sistema de drenaje se construya en aquellos túneles en los que se autorice el paso de mercancías peligrosas y cuando la longitud del túnel sea superior a 300 $\mathrm{m}$, que es la longitud del túnel a partir de la que se aplica dicha Circular. La Figura 6 muestra el sistema propuesto, el cual se compone de cuatro elementos fundamentales (Lingelser, 1994):

- Un sistema de recogida situado en el borde de la calzada, en la parte más baja del peralte (en los túneles se estudiará siempre poder desaguar la calzada hacia un solo lado), que el CETU propone sea un caz de sumidero continuo con entrada vertical.

- Sifones o arquetas cortafuegos cada $50 \mathrm{~m}$.

- Un colector general para evacuación desde los sifones o arquetas cortafuegos.

- Un depósito o balsa de retención en el exterior del túnel.

En la consideración de fuego generado por un vertido accidental, este elemento de seguridad instalado en los túneles mitigará los daños que pueda generar el mismo. Pese a ser costoso de construir y de mantenimiento importante, según lo que hemos visto, en los túneles de más de 500 m (según la Directiva Europea) o de 300 m (Francia) en los que se autorice el paso de mercancías peligrosas preceptivamente se han de construir.

\section{Conclusiones}

Un túnel supone un elemento singular en el conjunto de la red viaria y, como tal, requiere un tratamiento
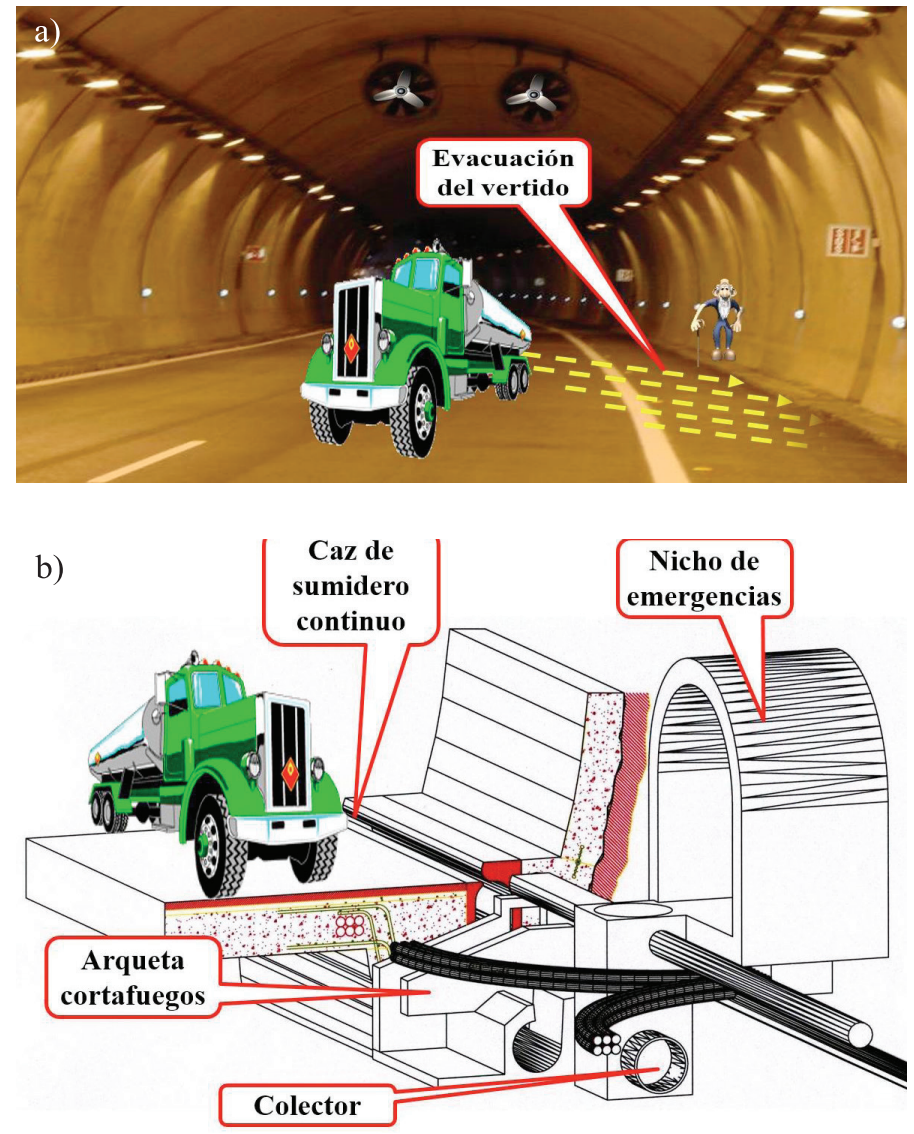

Figure 6: a) Evacuación del vertido en el túnel según la línea de máxima pendiente y b) sistema de recogida de vertidos accidentales situado en el borde de la calzada, en el lado bajo del peralte

especial de sus características que incremente y mejore, en la medida de lo posible, la seguridad del usuario. Los graves accidentes sufridos en diversos túneles europeos, con enorme repercusión social y pésimas consecuencias humanas, derivó en España en la publicación del Real Decreto 635 (2006) sobre requisitos mínimos de seguridad en los túneles de carreteras del Estado. Este, entre otras disposiciones, exige colocar pavimento de hormigón en los túneles de más de $1000 \mathrm{~m}$ de longitud (tengan o no tengan autorizado el paso de mercancías peligrosas), salvo razones que queden debidamente justificadas, $\mathrm{y}$ que nunca supongan una merma de la seguridad para el usuario en el interior del túnel. En el caso de túneles que tengan autorizado el paso de mercancías peligrosas, las normativas de Europa y de España exigen instalar un sistema de drenaje para vertidos accidentales en túneles de más de 500 m y más de 300 m en Francia.

En consonancia con la normativa de Europa y de España, 
los autores del presente artículo consideramos que el firme de hormigón se debe de exigir en los túneles de más de $500 \mathrm{~m}$ (y no $1000 \mathrm{~m}$ ) que tengan autorizado el paso de mercancías peligrosas (si nos referimos a la normativa de Francia, se debería exigir en túneles de más de 300 m).

La mayor contribución del pavimento de hormigón para proporcionar un elevado nivel de confort y seguridad en el túnel es ampliamente conocida, al ofrecer una superficie más clara y luminosa (que además permite reducir el gasto en iluminación), su facilidad para lograr una textura poco ruidosa y durable, el ahorro de combustible que conlleva el firme rígido no deformable o las mínimas operaciones requeridas de conservación, que reducen las afecciones al usuario y las posibilidades de cualquier accidente. Pero, es aún más importante, el aumento de seguridad que proporciona el pavimento de hormigón en el caso de un incendio provocado por cualquier trágico accidente. Frente a otras soluciones, se reduce la emisión de humos y gases tóxicos, no aumenta la carga de fuego, no es inflamable, por lo que no contribuyen a la rápida extensión del fuego, y se mantiene integro a las temperaturas usualmente alcanzadas, lo que permite el acceso de los equipos profesionales de extinción. La Asociación Profesional de Técnicos de Bomberos APTB de España recomienda el empleo de pavimentos de hormigón en todos los túneles. También se recomienda estudiar la alternativa de utilizar un firme claro, como se hizo con los revestimientos de hormigón blanco, por su importante ahorro en el consumo de energía eléctrica del alumbrado durante toda la vida del túnel.

\section{Referencias}

APTB-IECA (2013). Contribución del pavimento de hormigón a la seguridad en los incendios en túneles de carretera. Simulación y criterios de intervención. Asociación Profesional de Técnicos de Bomberos e Instituto Español del Cemento y sus Aplicaciones. https://www.ieca.es/Uploads/docs/Informe_bomberos.pdf

CI (2000). Circulaire Interministerielle $\mathrm{N}^{\mathrm{o}} 2000-63$ du 25 aout 2000 relative à la sécurité dans les tunnels du réseau routier national. Ministere de L'Equipement des Transports et du Logement, Ministere de l'Interieur, Republique Française
CI (2006). Circulaire Interministerielle $\mathrm{N}^{0} 2006-20$ du 29 mars 2006 relative à la sécurité des tunnels routier d'une longueur supérieure à 300 mètres. Ministere de L'Equipement des Transports et du Logement, Ministere de l'Interieur, Republique Française de Lathawer, W. (2007). Effects of pavement on fires in road tunnels. Routes: Revue de l'AIPCR 334, 54-61 del Val Melús, M.Á., Romana García, M. y Galvis, R. (2007). Una propuesta para el proyecto de firmes y pavimentos en túneles. Revista de Obras Públicas 3474, 7-18

Díaz, J. (2016). Más de 40 años del pavimento de hormigón armado continuo de la Y de Asturias. Rutas 169, 27-33

Díaz, J. (2010). Pavimentos eternos: actuaciones innovadoras de hace 35 años. "La Y de Asturias". Rutas 138, 11-19

Directiva (2004). Requisitos mínimos de seguridad para túneles de la red Europea de Carreteras. Directiva 2004/54/CE del Parlamento Europeo y del Consejo de la Unión Europea. Diario Oficial de la Unión Europea DOUE, 7 de junio de 2004

FCC Construcción (2010). El pavimento de hormigón regresa a las carreteras españolas. FCC realiza con éxito una demostración en las obras de la carretera C-17 en Barcelona. 26 de abril de 2010, http://www.fccco.com/es/-/el-pavimento-de-hormigonregresa-a-las-carreteras-espanolas

GIDAI (2011). Análisis experimental del comportamiento al fuego de pavimentos empleados en túneles de carretera. Grupo de Investigación y Desarrollo GIDAI, Universidad de Cantabria y ETS de Ingenieros Industriales y de Telecomunicación, España Jofré, C., Romero, J. y Rueda, R. (2010a). Consideraciones en torno al comportamiento del pavimento en la seguridad de los túneles frente al fuego. V Simposio de Túneles de Carretera: Seguridad para los Túneles del siglo XXI. Asociación Técnica de Carreteras ATC, Bilbao, España

Jofré, C., Romero, J. y Rueda, R. (2010b). Pavimentos de hormigón en túneles. Su influencia en la seguridad frente al fuego. Instituto Español del Cemento y sus Aplicaciones IECA, Madrid, España

Lacroix, D., Casale, E., Cwiklinski, C. and Thiboud, A. (1995). Full size testing of drainage systems for burning liquids in road tunnels. World Tunnel Congress and STUVA Stuttgart, Germany, 230-236 
Lingelser, S. (1994). Essais hydrauliques des systemes de recueil des liquides repandus sur la chaussee des tunnels routiers. Rapport de recherche. Centre d'Etudes des Tunnels CETU, Lyon, France

Miró, R. (2006). Nuevas mezclas para capas de rodadura y su influencia en el confort (ruido) y la seguridad. Universidad Politécnica de Cataluña, Zaragoza, España

Perard, M., Casale, E., Cwiklinski, C., Thiboud, A. and Deterne, J. (1996). Essais en vraie grandeur de systèmes de recueil des liquides enflammés répandus sus la chaussée d'un tunnel routier. Tunnels et Ouvrages Souterrains 135, 131-151

Real Decreto 635 (2006). Requisitos mínimos de seguridad en los túneles de carreteras del Estado. BOE de 27 de mayo de 2006. Corrección de errores en el BOE de 31 de julio de 2006. Ministerio de Fomento, Gobierno de España
Resolución (2012). Metodología de análisis de riesgo en túneles de la red de carreteras del estado. Resolución aprobada el 30 de mayo de 2012. Secretaría de Estado de Infraestructuras, Transporte y Vivienda. Dirección General de Carreteras. Subdirección General de Conservación. Ministerio de Fomento, Gobierno de España

Routes 83 (2003). Chaussée béton et tunnels: la juste equation. 7-12. Le tunnel de Cointe: un ouvrage exemplaire. Extraits du rapport du Ministère Wallon de l'Equipement et des Transports, $13-14$ 